INFECTIOUS DISEASE

\section{A new tool for testing malaria vaccines}

Vaccines directed against circumsporozoite (CS) proteins of Plasmodium falciparum (a causative agent of malaria) are undergoing Phase I human trials at present. One of the goals of these vaccines is to generate neutralizing antibodies specific for sporozoites (the pre-erythrocytic, blood-stage form of the parasite). But, $P$. falciparum does not infect rodents, and it is not possible to assess the efficacy of vaccine-induced neutralizing antibody responses in the lab. Now, researchers at New York University have developed an animal model for testing vaccine-induced antibody responses by modifying a rodent species of malaria.

Hybrid parasites were generated by targeting a CS-deficient form of the rodent malaria parasite Plasmodium berghei with a plasmid encoding $P$. falciparum $\mathrm{CS}$ repeat regions (CS(Pf)). To assess whether the modified rodent parasites would be useful for measuring protective antibody responses, mice were immunized with a vaccine containing $P$. falciparum $\mathrm{CS}$ repeats and then exposed to mosquitoes infected with the $\mathrm{CS}(\mathrm{Pf})$-modified

parasite. The vaccine generated sterile immunity in the immunized mice, and protection was specific for P. falciparum CS repeats. Sera from immunized mice protected naive recipients from infection when challenged with mosquitoes infected with CS(Pf)-modified parasites. Depletion of $\mathrm{CD}^{+} \mathrm{T}$ cells did not affect the level of protection of immunized mice, which indicates that protection was mediated predominantly by antibodies.

These CS(Pf)-modified rodent parasites are a powerful and safe new tool for the analysis of vaccineinduced antibody responses. By expressing T-cell epitopes from the $P$. falciparum CS protein, it should be possible to develop hybrid parasites for the analysis of T-cell responses.

Elaine Bell

Q) References and links ORIGINAL RESEARCH PAPER Persson, C. et al. Cutting edge: a new tool to evaluate human pre-erythrocytic malaria vaccines: rodent parasites bearing a hybrid Plasmodium falciparum circumsporozoite protein. J. Immunol. 169 6681-6685 (2002)

FURTHER READING Richie, T. L. \& Saul, A. Progress and challenges for malaria vaccines. Nature 415, 694-701 (2002)

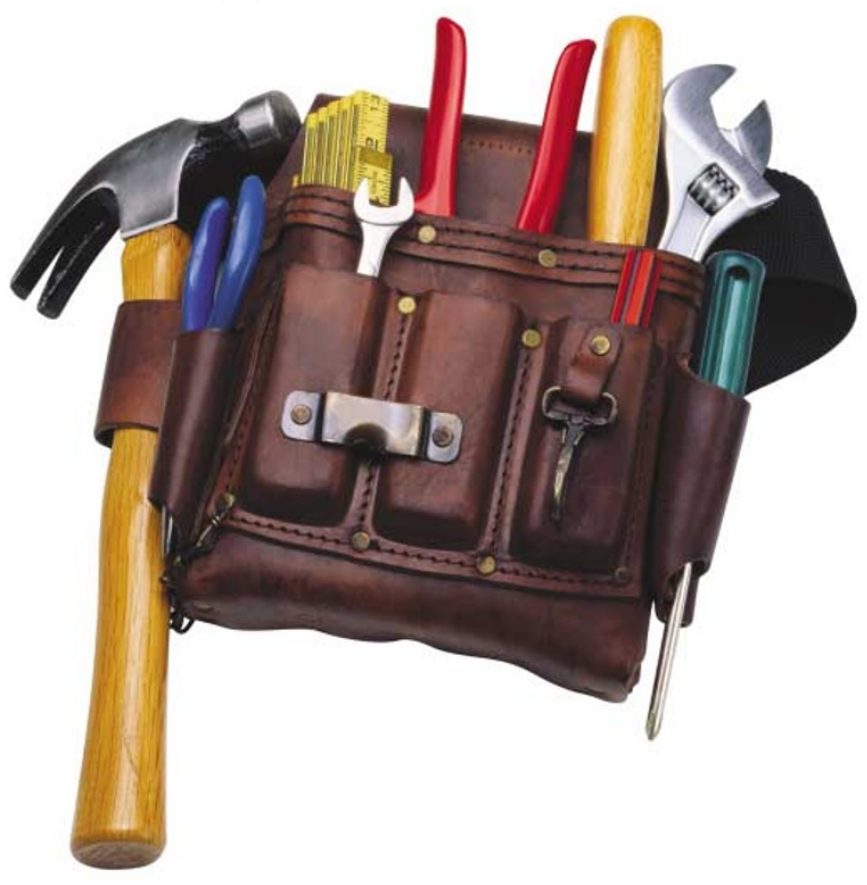

\section{IN BRIEF}

\section{LYMPHOID ORGANOGENESIS}

Different cytokines induce surface lymphotoxin- $\alpha \beta$ on IL-7 receptor- $\alpha$ cells that differentially engender lymph nodes and Peyer's patches.

Yoshida, H. et al. Immunity 17, 823-833 (2002)

The interleukin-7 receptor- $\alpha(\text { IL-7R } \alpha)^{+}$'inducer' cells for Peyer's patch (PP) and lymph node (LN) development are phenotypically similar, and both must be triggered to express lymphotoxin- $\alpha \beta(L T \alpha \beta)$ for their function. But, there are differences in the molecular requirements for PP and LN development. The development of PPs, but not LNs, requires IL-7, whereas LN development requires signals through RANK ligand (RANKL). This study shows that in Traf6 $6^{-1-}$ mice, in which RANKL signalling and LN development is blocked, IL-7 can stimulate the expression of LT $\alpha \beta$ on LN inducer cells and treatment with soluble IL-7 restores LN formation. This indicates that the inducer cells for LNs and PPs are functionally similar also.

\section{TRANSPLANTATION}

$\mathrm{CD} 4^{+} \mathrm{CD} 25^{+}$alloantigen-specific immunoregulatory cells that can prevent CD8 ${ }^{+}$T-cell-mediated graft rejection: implications for anti-CD154 immunotherapy.

van Maurik, A. et al. J. Immunol. 169, 5401-5404 (2002)

Blockade of the CD40-CD154 co-stimulatory pathway using CD154-specific antibodies can promote the long-term survival of allografts in animal models, and there is some evidence that this is mediated, in part, by $\mathrm{CD} 4^{+}$regulatory $\mathrm{T}$ cells. But, in some situations, anti-CD154 therapy fails owing to the initiation of graft rejection by CD154-independent $\mathrm{CD}^{+}$ $\mathrm{T}$ cells. This study shows that anti-CD154 treatment at the time of transplantation allows the development of $\mathrm{CD} 4^{+} \mathrm{CD} 25^{+}$ regulatory $\mathrm{T}$ cells, which, if present in sufficient numbers, can suppress rejection mediated not only by $\mathrm{CD} 4^{+} \mathrm{T}$ cells, but also by $\mathrm{CD} 8^{+} \mathrm{T}$ cells. This phenomenon, known as linked unresponsiveness, is an important clinical goal.

\section{ANTIBODY RESPONSES}

\section{A critical role for IL-21 in regulating immunoglobulin} production.

Ozaki, K. et al. Science 298, 1630-1633 (2002)

The authors generated IL-21 receptor (IL-21R)-deficient mice and found that although T-cell function was normal in these animals, the levels of IgG1 and IgG2b were reduced and the level of IgE was increased. However, this B-cell-intrinsic defect is less severe than that seen in mice and humans that are deficient for the cytokine-receptor common $\gamma$-chain (which is shared by IL-21R), indicating that normal B-cell responses must involve an additional cytokine(s). Mice that were deficient for both IL-21R and IL-4 had markedly impaired production of all antibody isotypes. So, this study shows that co-operation between IL-4 and IL-21 is essential for antibody responses. 